\title{
Time-Switching Energy Harvesting in Relay Networks
}

\author{
Saman Atapattu ${ }^{\dagger}$, Hai Jiang ${ }^{\ddagger}$, Jamie Evans ${ }^{\dagger}$, and Chintha Tellambura ${ }^{\ddagger}$ \\ $\dagger$ Department of Electrical and Computer Systems Engineering, Monash University, Australia \\ $\ddagger$ Department of Electrical and Computer Engineering, University of Alberta, Canada
}

\begin{abstract}
We consider a wireless energy harvesting (EH) relay network. Relays without embedded energy supply harvest energy from the source node. Considering a time switching protocol, performance measures such as average signal-to-noise ratio (SNR), outage and throughput are analyzed. Subsequently, optimal EH time is selected in order to maximize the throughput. Then, a multiple-relay network is considered with relay selection, which can achieve full diversity at any EH time. All theoretical results are validated by numerical simulations.
\end{abstract}

Index Terms-Energy harvesting, relay selection, outage probability, throughput.

\section{INTRODUCTION}

Wireless energy-harvesting $(\mathrm{EH})$ where devices collect energy from radio-frequency (RF) signals has recently received a lot of attention. EH can be performed simultaneously with information decoding, which has been considered in the original works [1], [2]. Since this protocol has implementation difficulties, a practically realizable protocol which processes EH ahead of information decoding has been motivated [3]-[5]. In the literature, $\mathrm{EH}$ has been considered for point-to-point communications [1]-[3] and cooperative relay communications [4], [6]-[13]. In relay networks, if additional relay nodes have limited power accessibility (as they can be randomly scattered mobile nodes in a network), EH can be a promising technique to energize the relay nodes.

In relay networks, $\mathrm{EH}$ techniques have been studied for decode-and-forward (DF) relaying in [6]-[10] and amplifyand-forward $(A F)$ relaying in [4], [11]-[13]. Source and relay nodes may obtain energy from energy-harvesting resources. There are three main EH methods: Method 1: source and relay nodes harvest energy from external sources such as solar, wind, or electromechanical energy [6], [7], [9], [11], [13]; Method 2: source node(s) has own fixed power supply, and relays are equipped with batteries which harvest energy from RF signals of source(s) [4], [8]; and Method 3: source and relay nodes have own fixed power supplies, and there is a separate $\mathrm{EH}$ receiver which allows low-powered wireless devices, e.g., in sensor networks, in its vicinity to utilize the harvested energy [10], [12]. For EH and information decoding, a receiver may have two sets of independent antennas or may share the same antenna(s). The latter needs less hardware complexity than the former, and can be implemented even with single antenna. When the same antenna(s) is shared, the time-switching architecture can be applied, in which a simpler switch selects either $\mathrm{EH}$ or information receiving at a time.

While EH relay networks which are implemented with a combination of the aforementioned techniques are analyzed for different objectives [6]-[11], in this paper, we analyze the EH Method 2 with an AF relaying network operated under a time-switching architecture because this combination may be a practically realizable setup. There is limited research work on this setup (or closely related setups) e.g., [4]. However, this setup results in a complicated end signal-to-noise ratio (SNR) expression which makes performance analysis intractable. To solve the problem, our paper provides the following contributions. i) In [4], outage probability is derived in a closedform only for high-SNR. Our paper drives the average endSNR and outage probability in closed-forms for any SNR, which are novel expressions, when source-to-relay and relayto-destination channels are Rayleigh and AWGN, respectively. ii) The EH time in a time-switching protocol is important due to its effect on the throughput. In [4], the optimal EH time is calculated numerically due to analytical complexity of the throughput expression. However, our throughput expression helps to derive the optimal $\mathrm{EH}$ time analytically. iii) Since a single-relay EH network may not achieve the performance of a single non-EH network (or traditional relay network) even at the optimal EH time, relay selection (RS) from multiple relays has been motivated. Although some RS schemes are proposed for simultaneous information transmission and power transfer [14], [15], RS is rarely investigated for the setup considered in this paper [16]. Thus, we propose an RS scheme that achieves full diversity and achieves throughput of non-EH network with properly selected EH time.

The rest of this paper is organized as follows. Section II discusses the system model. Section III analyzes a single-relay EH network. Section IV is for the analysis of RS in multiplerelay networks. Section V presents numerical and simulation results. Conclusion is given in Section VI.

\section{System Model}

This section describes time-switching communications protocol, analytical model, and fading channels.

Time-Switching Protocol: Consider a wireless relay network with a source $(S)$ sending information to its destination $(D)$ via a relay $(R)$, as shown in Fig. 1a. The source has a single antenna with power budget denoted as $P$, while the relay also has a single antenna with power budget denoted as $Q$. Assume direct link does not exist between the source and the destination. All channels are independent. For the channels from the source to the relay and from the relay to the destination, denote $f$ and $g$ as fading coefficients, respectively. ${ }^{1}$ Communications take place in half-duplex mode. The transmission block period is $T$. In contrast to the two-step communications protocol of the traditional relay network, the EH relay network follows three steps (which are also shown in Fig. 1b):

- Step 1: the relay harvests energy from the source's RF signals for $\alpha T$ time duration, where $0 \leq \alpha \leq 1$;

\footnotetext{
${ }^{1}$ In this paper, while the fading is considered, the path loss effect is neglected because we do not consider the effect of distance between nodes. However, following analysis is still valid with the path loss effect.
} 


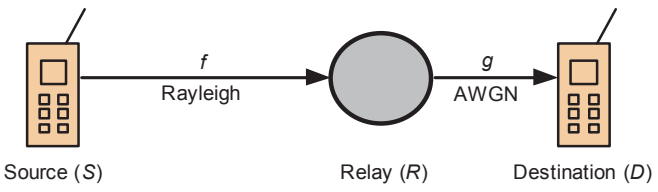

(a)

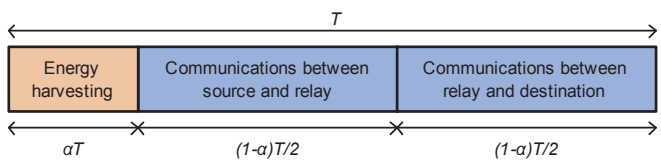

(b)

Fig. 1: (a) Single-relay network; (b) Three-step EH protocol.

- Step 2: the source communicates with the relay over (1a) $T / 2$ time duration;

- Step 3: the relay communicates with the destination over $(1-\alpha) T / 2$ time duration.

Analytical Model: Denote $s$ as the source's information symbol, with unit average energy. This symbol is transmitted for $\mathrm{EH}$ and for data transmission in the first and second steps, respectively. Thus, the received signal at the relay can be written as $y_{r}=\sqrt{P} f s+n_{r}$, where $n_{r}$ is the additive noise at the relay, which is assumed to be independent and identically distributed (i.i.d.) following complex Gaussian with zero-mean and $N_{0}$ variance, i.e., $n_{r} \sim \mathcal{C N}\left(0, N_{0}\right)$. The relay harvests energy for $\alpha T$ time duration using the RF signal $y_{r}$ with the rectification efficiency $\eta, 0<\eta<1$. Thus, the harvested energy at the relay is

$$
E=\eta P \alpha T|f|^{2}=\eta P \alpha T \gamma_{f}
$$

where $\gamma_{f}=|f|^{2}$. By assuming that the relay utilizes all harvested energy $E$ during the third step, the average transmit power of the relay can be given as $Q=\frac{2 \eta P \alpha \gamma_{f}}{1-\alpha}$. By assuming that the relay has knowledge of instantaneous channel state information (CSI) $f$, the coherent power coefficient of the $\mathrm{AF}$ relay is $G=\frac{1}{1+P|f|^{2}}$ [17]. Thus, the received signal at the destination is given as $y_{d}=\sqrt{G Q} y_{r} g+n_{d}$ where $n_{d}$ is the additive noise at the destination with $n_{d} \sim \mathcal{C N}\left(0, N_{0}\right)$. Thus, the end-to-end receive SNR, which is called end-SNR, can be calculated as

$$
\gamma_{d}=\frac{\beta \rho^{2} \gamma_{f}^{2} \gamma_{g}}{\beta \rho \gamma_{f} \gamma_{g}+\rho \gamma_{f}+1}
$$

where $\beta=\frac{2 \eta \alpha}{1-\alpha}, \rho=\frac{P}{N_{0}}$, and $\gamma_{g}=|g|^{2}$. Since $P$ and $N_{0}$ are the average transmit power and noise power, respectively, we name $\rho$ as average $S N R$. In contrast to the end-SNR expression of a traditional relay network, i.e., $\gamma_{d}=\frac{\rho^{2} \gamma_{f} \gamma_{g}}{\rho \gamma_{f}+\rho \gamma_{g}+1}$, (2) has a complicated form when $\gamma_{f}$ and $\gamma_{g}$ have random distributions due to fading. Thus, it is important to use appropriate channel models which can i) represent valid physical network scenarios, and ii) help to develop an analytically tractable theoretical framework.

Fading Channels: A relay network has two channels in two hops, $S-R$ and $R-D$, which may have similar impact on the overall performance of a traditional relay network (non-EH). However, in an EH relay network, the first-hop channel, $S-R$, has a major impact on the network performance due to the following reasons [18]: 1) As (1), the harvested energy level at the relay depends only on the $S-R$ channel $f ; 2$ ) The coherent power coefficient $G$ and average transmit power $Q$ of the relay depend only on $f$; 3 ) EH is suitable for small scale networks, especially when the relay operates at a near distance to the destination. This is because, with EH method 2, we cannot expect to harvest a larger amount of energy which is sufficient for a larger distance and/or a longer time transmission. Further, the relay can be a small node which may not be able to handle a higher power level and/or may not have sufficient space. Thus, it is reasonable to locate the relay close to the destination in order to minimize the path loss/fading effect of the $R-D$ channel.

Based on these facts and analytical simplicity, we assume that $S-R$ and $R-D$ channels are Rayleigh and AWGN, respectively. Although the assumption of an unfaded $R-D$ link simplifies the analysis, the analytical results help us discuss EH more deeply as shown in following sections ${ }^{2}$.

\section{Analysis of A Single-Relay EH Network}

In this section, we analyze average end-SNR, outage probability, and throughput. Using these performance measures, we then analyze the optimal EH time.

\section{A. Performance Measures}

1) Average end-SNR: The instantaneous end-SNR is given in (2). With respect to (w.r.t.) the $S-R$ channel $f$, the average end-SNR $\bar{\gamma}_{d}$ can be given as $\bar{\gamma}_{d}=\int_{0}^{\infty} \gamma_{d} f_{\gamma_{f}}\left(\gamma_{f}\right) d \gamma_{f}\left(f_{\gamma_{f}}(\cdot)\right.$ is the probability density function of $\gamma_{f}$ ), leading to

$$
\begin{aligned}
\bar{\gamma}_{d}=\frac{\rho \bar{\gamma}_{f} \beta \bar{\gamma}_{g}}{\left(\beta \bar{\gamma}_{g}+1\right)}[1- & \frac{1}{\rho \bar{\gamma}_{f}\left(\beta \bar{\gamma}_{g}+1\right)} \\
& \left.-\frac{e^{\frac{\bar{\gamma}_{f}\left(\beta \bar{\gamma}_{g}+1\right)}{}} \operatorname{Ei}\left(-\frac{1}{\rho \bar{\gamma}_{f}\left(\beta \bar{\gamma}_{g}+1\right)}\right)}{\left[\rho \bar{\gamma}_{f}(\beta \gamma+1)\right]^{2}}\right]
\end{aligned}
$$

where $\operatorname{Ei}(\cdot)$ is the exponential integral function [19]. Further, $\bar{\gamma}_{g}=\mathbb{E}\left[|g|^{2}\right]=|g|^{2}$ as $R-D$ is AWGN, and $\mathbb{E}[\cdot]$ is the expectation operation. We omit the proof due to the space limitation.

2) Outage probability: The outage probability, denoted as $P_{\text {out }}$, is an important performance measure for communication systems. An outage means the received SNR is less than a predetermined threshold $\gamma_{t h}$. Thus, $P_{\text {out }}$ reflects the rate of successful transmission. The outage probability of the EH relay network, $P_{\text {out }}=\operatorname{Prob}\left[\gamma_{d}<\gamma_{t h}\right]$ (Prob[.] means probability), can be calculated as

$$
P_{\text {out }}=1-e^{-\frac{1}{\rho \gamma_{f}}\left(\frac{\gamma_{t h}(1+\beta \bar{\gamma} g)+\sqrt{\gamma_{t h}^{2}(1+\beta \bar{\gamma} g)^{2}+4 \beta \bar{\gamma} g \gamma_{t h}}}{2 \beta \bar{\gamma}_{g}}\right)}
$$

where $\bar{\gamma}_{f}=\mathbb{E}\left[|f|^{2}\right]$. The proof is given in Appendix A.

3) Throughput: The throughput reflects the rate of successful transmission and efficiency of data transmission, which can be defined as [4]

$$
\tau=\frac{R(1-\alpha)\left(1-P_{\text {out }}\right)}{2} .
$$

We assume $R=1 \mathrm{bits} / \mathrm{sec} / \mathrm{Hz}$ in the rest of the paper, and thus, $\tau$ also means throughput efficiency.

\footnotetext{
${ }^{2}$ In [4], $R-D$ is assumed to be Rayleigh fading. However, performance is analyzed based on high-SNR assumption due to analytical difficulties.
} 


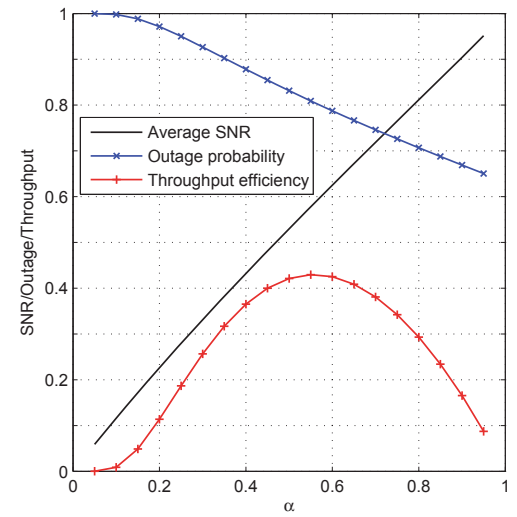

Fig. 2: Average end-SNR, outage and throughput efficiency with $\alpha$ for $\rho=\bar{\gamma}_{f}=\bar{\gamma}_{g}=\gamma_{t h}=0 \mathrm{~dB}$ and $\eta=1$.

Fig. 2 shows average end-SNR, outage probability and throughput w.r.t. $\alpha$ for $\rho=\bar{\gamma}_{f}=\bar{\gamma}_{g}=\gamma_{t h}=0 \mathrm{~dB}$ and $\eta=1$. When $\alpha \rightarrow 0$, we have worse performance in terms of all measures, i.e., $\bar{\gamma}_{d} \rightarrow 0, P_{\text {out }} \rightarrow 1$ and $\tau \rightarrow 0$, because no communication between the relay and destination is possible as no energy is harvested $(E \rightarrow 0)$. When $\alpha \rightarrow 1$, we have the best average end-SNR $\left(\bar{\gamma}_{d} \rightarrow \rho \bar{\gamma}_{f} \approx 1\right)$ and outage probability $\left(P_{\text {out }} \rightarrow 1-e^{-\frac{\gamma_{t h}}{\rho \gamma_{f}}} \approx 0.6321\right)$. At the same time, when $\alpha \rightarrow 1$, we have worse throughput because the relay harvests energy over the whole block time duration $T$, and the signal is forwarded to the destination with infinitely large power within infinitesimal time. Since the data throughput is limited by the channel bandwidth (which is not infinite), no data can be transmitted in infinitesimal time. Therefore, the throughput approaches 0 even though the end-SNR approaches to its maximum. Unlike a traditional relay network, the throughput efficiency in an EH relay network does not increase with endSNR. There is a maximum possible $\tau$ for a particular $\alpha$, e.g., the maximum possible throughput efficiency is $42 \%$ when $\alpha \approx 0.55$. This is the optimal $\alpha$ which maximizes $\tau$. This is discussed next in detail.

\section{B. Optimal EH Time}

The throughput is a function of $\alpha$ and $P_{\text {out }}$. When $\alpha$ increases (decreases), $P_{\text {out }}$ decreases (increases). Thus, as from (5), we can find the maximum $\tau$ in $\alpha \in(0,1)$. This corresponding $\alpha$ is called the optimal $\alpha$, and is denoted $\alpha^{*}$. Then the optimal EH time is $\alpha^{*} T$.

The value $\alpha$ decides the harvested energy $E$ at the relay. The optimal value $\alpha^{*}$ decides the energy level at the relay, which maximizes the throughput irrespective of the power level $Q$ at the relay. For example, when the $S-R$ channel is weak, the energy harvester may take longer time to accumulate sufficient energy for $R-D$ communications in order to achieve the maximum possible throughput. In this case, the relay utilizes a higher power level during shorter transmission time. On the other hand, when the $S-R$ channel is stronger, the energy harvester may take shorter time to accumulate sufficient energy, and then the relay utilizes a lower power level for longer data transmission time for maximum throughput. By assuming that the relay can adjust it power level to any value, the optimal value of $\alpha$ can be evaluated by using

$$
\alpha^{*}=\max _{\alpha} \frac{(1-\alpha)\left(1-P_{\text {out }}\right)}{2}, \text { where } 0<\alpha<1 .
$$

An approximated value for the optimal $\alpha$ can be calculated as

$$
\alpha_{k}^{*} \approx-\frac{1}{3}\left(b+u_{k} C+\frac{\Delta_{0}}{u_{k} C}\right), \quad k \in 1,2,3
$$

where $u_{1}=1, u_{2}=\frac{i \sqrt{3}-1}{2}$ ( $i$ being imaginary unit), $u_{3}=$ $\frac{-i \sqrt{3}-1}{2}, C=\left(\frac{\Delta_{1}+\sqrt{\Delta_{1}^{2}-4 \Delta_{0}^{3}}}{2}\right)^{\frac{1}{3}}, \Delta_{0}=b^{2}-3 c$, and $\Delta_{1}=$ $2 b^{3}-9 b c+27 d$. Further, $b=\frac{2 \eta\left(1+\bar{\gamma}_{f} \rho+\gamma_{t h}\right) \bar{\gamma}_{g}-\gamma_{t h}}{2 \eta \bar{\gamma}_{f} \rho \bar{\gamma}_{g}\left(2 \eta \bar{\gamma}_{g}-1\right)}, c=$ $\frac{2 \gamma_{t h}-2 \eta\left(1+\gamma_{t h}\right) \bar{\gamma}_{g}}{2 \eta \bar{\gamma}_{f} \rho \bar{\gamma}_{g}\left(2 \eta \bar{\gamma}_{g}-1\right)}$, and $d=\frac{\gamma_{t h}}{2 \eta \bar{\gamma}_{f} \rho \bar{\gamma}_{g}\left(1-2 \eta \bar{\gamma}_{g}\right)}$. The proof is given in Appendix B. The optimal value $\alpha^{*}$ is the one among $\alpha_{k}^{*}$ in (7) which satisfies $0<\alpha_{k}^{*}<1$ and has maximal $\tau\left(\alpha_{k}^{*}\right)$. The accuracy of the approximation is shown by the $\alpha^{*}$ vs. SNR $\rho$ and throughput vs. SNR $\rho$ curves in Figs. $4 \mathrm{a}$ and $4 \mathrm{~b}$, respectively (see discussion in Section V).

So far we consider a single-relay EH network, and how maximum throughput can be achieved with optimal EH time. As explained in Section III-A, a single-relay EH network can approach the throughput (i.e., 0.5) of a traditional singlerelay network, i.e., $\tau \rightarrow 0.5$ with a high power $P$ (i.e., high $\rho$ ). For example, as to be shown in Fig. $4 \mathrm{~b}, 80 \%$ of throughput efficiency of a traditional (non-EH) single-relay network can be achieved when $\rho>17 \mathrm{~dB}$ at $\gamma_{t h}=0 \mathrm{~dB}$. However, such high $\rho$ may not be available with moderate transmission power $P$ in a high noise power environment. Further, high transmission power $P$ may not be feasible in practice. Therefore, as an alternative, we consider a multiplerelay network, because such networks can provide diversity advantage, to further increase the throughput. This is discussed in the next section.

\section{Single RS in Multiple-Relay EH Network}

Instead of one relay as shown in Fig. 1a, we consider a relay network with $N$ intermediate relays. For the $i$ th relay, its power budget is denoted $Q_{i}$, and the fading coefficients from the source to itself and from itself to the destination are $f_{i}$ and $g_{i}$, respectively. All channels are independent. The end-SNR via the $i$ th relay of the EH network can be given as

$$
\gamma_{d_{i}}=\frac{\beta_{i} \rho_{i}^{2} \gamma_{f i}^{2} \gamma_{g i}}{\beta_{i} \rho_{i} \gamma_{f i} \gamma_{g i}+\rho_{i} \gamma_{f i}+1}
$$

where $\beta_{i}=\frac{2 \eta_{i} \alpha_{i}}{1-\alpha_{i}}, \rho_{i}=\frac{P}{N_{0, i}}, \gamma_{f i}=\left|f_{i}\right|^{2}$, and $\gamma_{g i}=\left|g_{i}\right|^{2}$. Here $\alpha_{i} T, \eta_{i}$ and $N_{0, i}$ are EH time duration, rectification efficiency and noise at the $i$ th relay. For simplicity, we assume $\eta_{i}=\eta$ and $N_{0, i}=N_{0}, \forall i$. Further, we assume $\alpha_{i}=\alpha$, $\forall i$ which avoids the synchronization problem at the relaying stage.

In traditional multiple-relay networks, original channels are assigned to relays (e.g., by frequency or time division multiple access) to avoid interference [20], which however greatly drops the throughput efficiency. Further, the EH process also requires $\alpha T$ of the transmission block, which further reduces 
throughput efficiency (as discussed in Section III). On the other hand, when multiple relays participate in forwarding the signal, the destination receives multiple copies of the transmitted signal, which greatly increases the complexity of synchronization. Therefore, with this particular EH protocol, it may not be a good choice to let all relays (or more than one relay) participate in the signal forwarding. This motivates single RS, which is discussed in the following.

In traditional relay networks (without $\mathrm{EH}$ ), the best relay is selected to achieve lowest outage/error rate or highest $\mathrm{SNR} /$ throughput. For example, the best relay, denoted as $i^{*}$ th relay, may be the relay with the highest end-SNR, i.e., $i^{*}=\arg \max _{i} \gamma_{d_{i}}$, where $\gamma_{d_{i}}$ is end-SNR via $i$ th relay. In EH relay networks, as we discussed in Section III-A, the highest end-SNR is possible when $\alpha \rightarrow 1$, but this gives worse throughput. Therefore, by considering the additional parameter $\alpha$, we extend the idea of $\mathrm{RS}$ in $\mathrm{EH}$ networks.

For any given $\alpha$, we denote the corresponding SNR in (8) as $\gamma_{d_{i}}(\alpha)$, and select the best relay among the available $N$ relays based on end-SNRs as $i^{*}=\arg \max _{i} \gamma_{d_{i}}(\alpha) .{ }^{3}$ By using the properties of order statistics, when channels are independent, the outage probability can be given as

$$
P_{\text {out }}=\prod_{i=1}^{N} P_{\text {out }, i}
$$

where $P_{\text {out }, i}=1-e^{-\frac{1}{\gamma_{f i}}\left(\frac{\gamma_{t h}\left(1+\beta \gamma_{g i}\right)+\sqrt{\gamma_{t h}^{2}\left(1+\beta \gamma_{g i}\right)^{2}+4 \beta \gamma_{g i} \gamma_{t h}}}{2 \beta \rho \gamma_{g i}}\right)}$ which is the outage probability via $i$ th relay, and $\bar{\gamma}_{f i}=\mathbb{E}\left[\gamma_{f i}\right]$. If channels are i.i.d., we can write $P_{\text {out }}=\left(P_{\text {out }, i}\right)^{N}$.

It is also important to investigate the achievable diversity order in the high SNR region. Diversity order, which is defined as $d \triangleq-\lim _{P \rightarrow \infty} \frac{\log P_{\text {out }}}{\log P}$ [21], shows the decrease speed of the outage probability when the transmit power $P$ increases in the high transmit power region (or high SNR region). For i.i.d. channels with $\bar{\gamma}_{f i}=\gamma_{g i}=\bar{\gamma}$, (9) can be written for the high transmit power region, i.e., $\rho \rightarrow \infty$, as

$$
\begin{aligned}
P_{\text {out }}=\left(\frac{1}{\rho}\right)^{N} \prod_{i=1}^{N} & {\left[\frac{\sqrt{\gamma_{t h}\left[4 \beta_{i} \bar{\gamma}+\gamma_{t h}\left(1+\beta_{i} \bar{\gamma}\right)^{2}\right]}}{2 \beta_{i} \bar{\gamma}^{2}}\right.} \\
& \left.+\frac{\gamma_{t h}\left(1+\beta_{i} \bar{\gamma}\right)}{2 \beta_{i} \bar{\gamma}^{2}}\right]+\mathcal{O}\left(\frac{1}{\rho}\right)^{N+1}
\end{aligned}
$$

which implies that RS can achieve diversity order $N$ at any given $\alpha$. The proof is omitted due to space limitation.

Since full diversity order can be achieved for any given $\alpha$, it is important to find a better $\alpha$ which can further improve the throughput efficiency. Therefore, we consider the throughput as a function of $\alpha$

$$
\tau(\alpha)=\frac{(1-\alpha)\left(1-\prod_{i=1}^{N} P_{\text {out }, i}\right)}{2}
$$

which gives the throughput efficiency when the best endSNR relay is selected for a given $\alpha T$ EH time. For example,

\footnotetext{
${ }^{3}$ This scheme is sub-optimal in maximizing throughput. The optimal scheme may be $i^{*}=\arg \max _{i} \tau_{i}\left(\alpha_{i}^{*}\right)$ where $\alpha_{i}^{*}$ is optimal EH time of $i$ th relay.
}

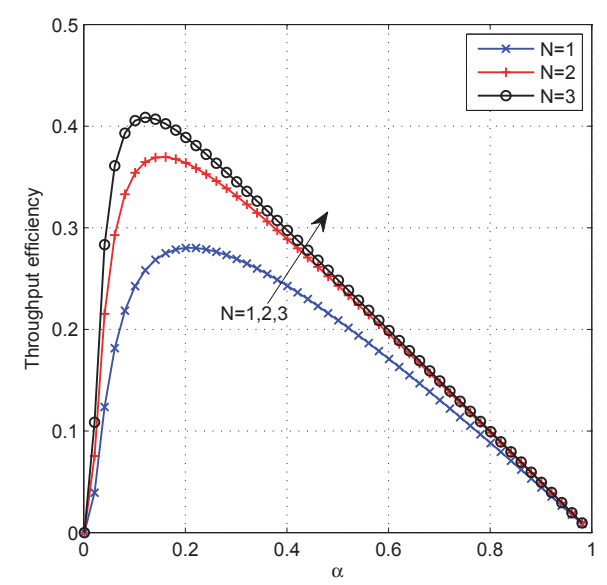

Fig. 3: Average throughput efficiency with $\alpha$ for $N=1,2,3$ for $\rho=\bar{\gamma}_{f i}=\gamma_{g i}=\gamma_{t h}=0 \mathrm{~dB}$ and $\eta=1$.

Fig. 3 shows throughput efficiency with $\alpha$ for different $N$ for $\rho=\bar{\gamma}_{f i}=\gamma_{g i}=\gamma_{t h}=0 \mathrm{~dB}$ and $\eta=1$. For any given $\alpha$, we can see that higher throughput efficiency can be achieved when the number of relays increases, e.g., at $\alpha=0.20$, $\tau=0.280,0.364,0.389$ for $N=1,2,3$, respectively. Further, there is a maximum throughput efficiency for each case, e.g., for $N=2, \tau=0.370$ at $\alpha=0.16$. This can be calculated as

$$
\hat{\alpha}=\underset{0 \leq \alpha \leq 1}{\operatorname{maximize}} \tau(\alpha)
$$

which is the best value of maximizing $\tau(\alpha)$ in (11). An approximate value for $\hat{\alpha}$ may also be calculated. However, in the rest of this paper, whenever necessary, we find $\hat{\alpha}$ by using numerical methods.

\section{Numerical and Simulation Results}

This section provides numerical results based on our analysis in Sections III and IV, and semi-analytical Monte-Carlo simulation results based on the system model in Section II.

For a single-relay EH network, this work derives an analytical approximation for the optimal EH time $\alpha^{*}$. Fig. $4 \mathrm{a}$ shows the simulated exact $\alpha^{*}$ and numerically calculated approximated $\alpha^{*}$ in (7) versus $\operatorname{SNR} \rho$, for $\gamma_{t h}=0 \mathrm{~dB}, 5 \mathrm{~dB}$, or $10 \mathrm{~dB}$ when $\eta=1$ and $\bar{\gamma}_{f}=\bar{\gamma}_{g}=0 \mathrm{~dB}$. The exact results (represented by solid curves) match tightly with the approximation results (represented by discrete marks) for the simulated SNR $\rho$ range at $\gamma_{t h}=5 \mathrm{~dB}$, or $10 \mathrm{~dB}$, while the other case $\left(\gamma_{t h}=0 \mathrm{~dB}\right)$ also shows close matches, confirming the validity of the approximation. However, the important point is that how this approximation affects the throughput value which is our main performance measure. We use approximated $\alpha^{*}$ to calculate the optimal throughput using (5). Fig. $4 \mathrm{~b}$ shows the optimal throughput efficiency vs. SNR $\rho$ for same parameters considered in Fig. 4a. For all cases, the exact results (solid curves) match tightly with approximation results (discrete marks) for the simulated SNR $\rho$ range, also confirming the validity of the approximation. Further, when the threshold increases, the throughput decreases because transmission is successful if receive end-SNR is large enough. 


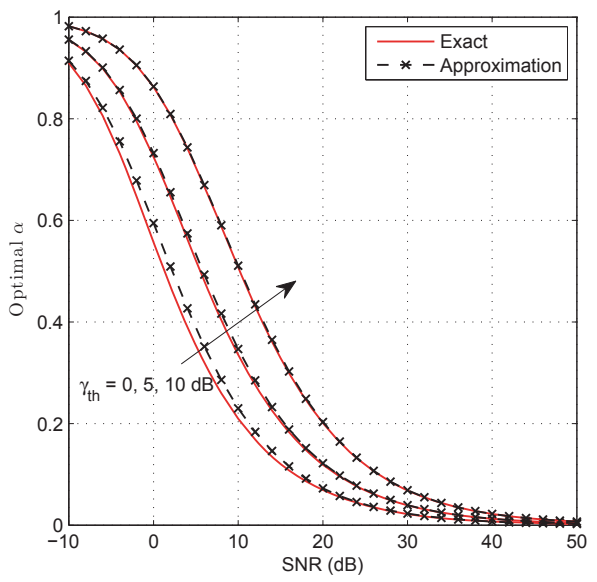

(a)

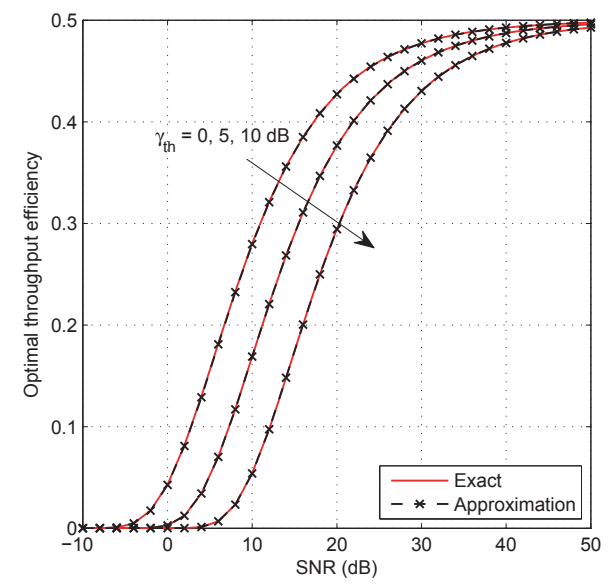

(b)

Fig. 4: (a) Optimal $\alpha$; (b) Throughput at optimal $\alpha$.

For our RS scheme in a multiple-relay EH network, its outage and throughput are illustrated in Fig. 5 comparing with the random $\mathrm{RS}$, in which the source randomly chooses a relay. Parameters are $N=2,3, \gamma_{t h}=0 \mathrm{~dB}, \eta=1$ and $\bar{\gamma}_{f}=\bar{\gamma}_{g}=0 \mathrm{~dB}$. For fixed harvesting time, i.e., $\alpha=0.3$, Fig. 5a shows outage versus SNR $\rho$. While our RS scheme has diversity order 2 and 3 when $N=2$ and $N=3$, respectively, which is full diversity order, the random RS has diversity order 1 which is the same as a single-relay network (i.e., $N=1$ ). Fig. 5b shows throughput efficiency versus $\operatorname{SNR} \rho$ when $\alpha=0.3$ and when $\alpha$ is selected according to (12). When $\alpha$ is fixed, the maximum achievable throughput (i.e., $\rho \rightarrow \infty$ ) can be derived by using (11) as $(1-\alpha) / 2$. With fixed $\alpha=0.3$, the maximum achievable throughput is 0.35 when $N=2$ or 3, which does not depend on $N$. With properly selected $\alpha$ according to (12), our RS scheme can achieve maximum throughput 0.5 , which is equal to throughput of traditional relay network (without $\mathrm{EH}$ ). This $\alpha$ selection even helps to improve performance of the random RS. However, for example

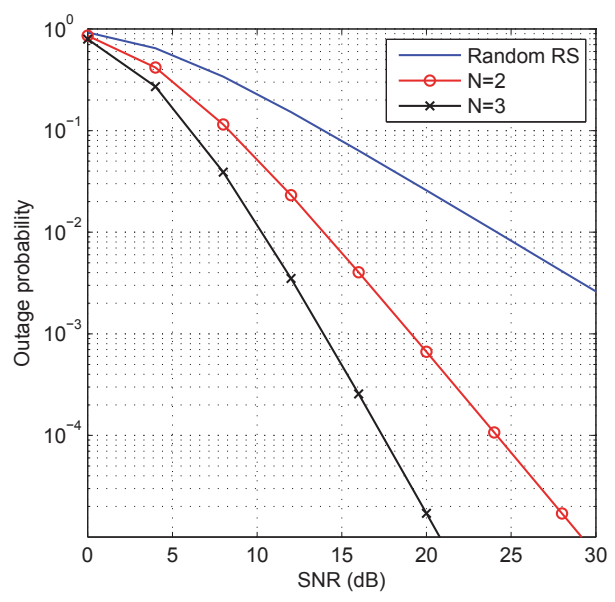

(a)

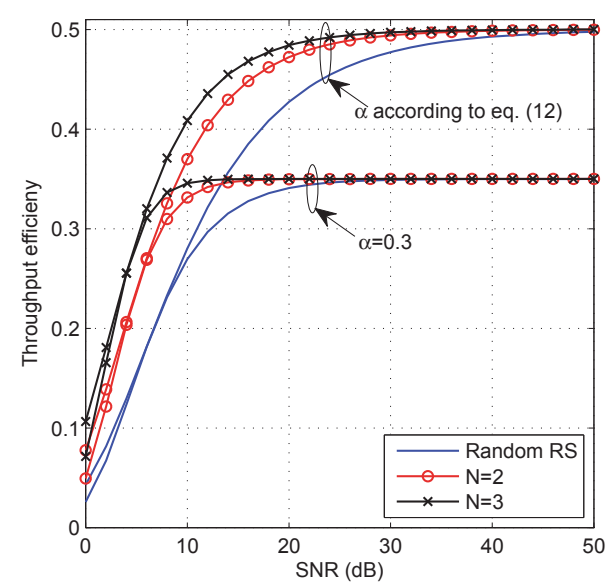

(b)

Fig. 5: (a) Outage probability when $\alpha=0.3$; (b) Throughput when $\alpha=0.3$ and $\alpha$ is selected as (12).

at $\rho=10 \mathrm{~dB}$, our RS can achieve around $75 \%$ and $80 \%$ of throughput of the traditional relay network when $N=2,3$, respectively, but random RS (or single-relay network) only achieves around $58 \%$. So proper RS and $\alpha$ selection are important in $\mathrm{EH}$.

\section{CONCLUSION}

For a time-switching EH relay network in which the relay is energized by the source's RF signals, closed-form expressions for average SNR, outage and throughput are derived when the first and second hop channels are Rayleigh fading and Gaussian, respectively. Then, an approximation for the optimal EH time is derived that maximizes throughput. Subsequently, for multiple-relay EH network, a single RS scheme is proposed, which achieves full diversity at any $\mathrm{EH}$ time. $\mathrm{EH}$ time when $\mathrm{RS}$ is applied is also proposed to achieve 50\% throughput efficiency (which is equal to throughput efficiency in non-EH relay networks) at high SNR. 


\section{APPENDIX}

\section{A. Proof of (4)}

$$
\begin{aligned}
P_{\text {out }} & =\operatorname{Prob}\left[\gamma_{d}<\gamma_{t h}\right]=\operatorname{Prob}\left[\frac{\beta \rho^{2} \gamma_{f}^{2} \gamma_{g}}{\beta \rho \gamma_{f} \gamma_{g}+\rho \gamma_{f}+1}<\gamma_{t h}\right] \\
& =\operatorname{Prob}\left[\left(\beta \rho^{2} \gamma_{g}\right) \gamma_{f}^{2}-\gamma_{t h} \rho\left(1+\beta \gamma_{g}\right) \gamma_{f}-\gamma_{t h}<0\right] \\
& =\operatorname{Prob}\left[\left(\gamma_{f}-\gamma_{1,1}\right)\left(\gamma_{f}-\gamma_{1,2}\right)<0\right]
\end{aligned}
$$

where $\gamma_{1,1}=\frac{\gamma_{t h}\left(1+\beta \gamma_{g}\right)+\sqrt{\gamma_{t h}^{2}\left(1+\beta \gamma_{g}\right)^{2}+4 \beta \gamma_{g} \gamma_{t h}}}{2 \beta P \gamma_{g}}, \gamma_{1,2}=$ $\frac{\gamma_{t h}\left(1+\beta \gamma_{g}\right)-\sqrt{\gamma_{t h}^{2}\left(1+\beta \gamma_{g}\right)^{2}+4 \beta \gamma_{g} \gamma_{t h}}}{2 \beta P \gamma_{g}}$ and $\gamma_{1,1} \geq 0, \gamma_{1,2} \leq 0$. Since $\gamma_{f} \geq 0$ and the inequality $\left(\gamma_{f}-\gamma_{1,1}\right)\left(\gamma_{f}-\gamma_{1,2}\right)<0$ satisfies when $\gamma_{1,2}<\gamma_{f}<\gamma_{1,1}$, $P_{\text {out }}$ in (13) can be given as

$$
P_{\text {out }}=\operatorname{Prob}\left[0<\gamma_{f}<\gamma_{1,1}\right]=F_{\gamma_{f}}\left(\gamma_{1,1}\right)
$$

where the $\mathrm{CDF}$ of $\gamma_{f}$ can be given as $F_{\gamma_{f}}(\gamma)=1-e^{-\frac{\gamma}{\gamma_{f}}}$, which proves (4).

\section{B. Proof of (7)}

For the optimal $\alpha, \alpha^{*}=\arg \max \tau(\alpha)$ is achieved when $\frac{\partial \tau(\alpha)}{\partial \alpha}=0$. With the aid of (4) and (5), we can write

$$
\begin{aligned}
f(\alpha)= & (\alpha-1)\left(\alpha-1-2 \eta \bar{\gamma}_{g} \alpha\right) \gamma_{t h}^{2}-4 \eta \bar{\gamma}_{g} \alpha \gamma_{t h}(\alpha-1) \\
& +\left[4 \eta \bar{\gamma}_{f} \rho \bar{\gamma}_{g} \alpha^{2}+(\alpha-1) \gamma_{t h}\right] \sqrt{T_{N}}=0
\end{aligned}
$$

where $T_{N}=\gamma_{t h}^{2}\left(\alpha-1-2 \eta \bar{\gamma}_{g} \alpha\right)^{2}-8 \gamma_{t h} \eta \bar{\gamma}_{g} \alpha(\alpha-1)$. Since it is difficult to find an exact solution for the term $\sqrt{T_{N}}$, we use the following approximation on $T_{N}$ :

$$
\begin{aligned}
& T_{N}=\gamma_{t h}^{2}\left[1-2 \alpha+4 \eta \bar{\gamma}_{g} \alpha+\alpha^{2}-4 \eta \bar{\gamma}_{g} \alpha^{2}\right. \\
& \left.+4 \eta^{2} \bar{\gamma}_{g}^{2} \alpha^{2}+\frac{8 \eta \bar{\gamma}_{g} \alpha(1-\alpha)}{\gamma_{t h}}\right] \\
& \quad \stackrel{(a)}{\approx} \gamma_{t h}^{2}\left(1-2 \alpha+4 \eta \bar{\gamma}_{g} \alpha+\alpha^{2}-4 \eta \bar{\gamma}_{g} \alpha^{2}+4 \eta^{2} \bar{\gamma}_{g}^{2} \alpha^{2}\right) \\
& =\gamma_{t h}^{2}\left(-1+\alpha-2 \eta \bar{\gamma}_{g} \alpha\right)^{2}
\end{aligned}
$$

where $(a)$ comes by neglecting the term $\frac{8 \eta \bar{\gamma}_{g} \alpha(1-\alpha)}{\gamma_{t h}}$ comparing the values of other terms. This can be justified because $\alpha(1-\alpha) \leq 0.25$ for $0<\alpha<1$, and also we can assume $\gamma_{t h}>1$. Thus, the contribution of term $\frac{8 \eta \bar{\gamma}_{g} \alpha(1-\alpha)}{\gamma_{t h}}$ is neglected. Then, (15) can be approximated as

$$
\begin{aligned}
f(\alpha) \approx & \gamma_{t h}\left[2 \gamma_{t h}(1-\alpha)\left(1+2 \eta \bar{\gamma}_{g} \alpha-\alpha\right)\right. \\
& \left.+4 \eta \bar{\gamma}_{g} \alpha\left(1-\alpha-\bar{\gamma}_{f} \rho \alpha+\bar{\gamma}_{f} \rho \alpha^{2}-2 \bar{\gamma}_{f} \rho \eta \bar{\gamma}_{g} \alpha^{2}\right)\right] .
\end{aligned}
$$

Since we need the optimal value, $\alpha^{*}$, it can be found approximately by solving $f(\alpha)=0$ for $\alpha$ by using (16). This can be written as a polynomial in $\alpha$ with degree 3 as

$$
\begin{aligned}
\alpha^{3} & +\frac{2 \eta\left(1+\bar{\gamma}_{f} \rho+\gamma_{t h}\right) \bar{\gamma}_{g}-\gamma_{t h}}{2 \eta \bar{\gamma}_{f} \rho \bar{\gamma}_{g}\left(2 \eta \bar{\gamma}_{g}-1\right)} \alpha^{2} \\
& +\frac{2 \gamma_{t h}-2 \eta\left(1+\gamma_{t h}\right) \bar{\gamma}_{g}}{2 \eta \bar{\gamma}_{f} \rho \bar{\gamma}_{g}\left(2 \eta \bar{\gamma}_{g}-1\right)} \alpha-\frac{\gamma_{t h}}{2 \eta \bar{\gamma}_{f} \rho \bar{\gamma}_{g}\left(2 \eta \bar{\gamma}_{g}-1\right)}=0 .
\end{aligned}
$$

By using the above cubic equation, the general solution for the roots is given in (7). Further, Any cubic equation can be exactly solved in closed-form by using results in the literature (e.g., [22]) or the built-in functions in software packages.

\section{REFERENCES}

[1] L. Varshney, "Transporting information and energy simultaneously," in Proc. IEEE Int. Symp. Inf. Theory (ISIT), Jul. 2008, pp. 1612-1616.

[2] P. Grover and A. Sahai, "Shannon meets Tesla: Wireless information and power transfer," in Proc. IEEE ISIT 2010, pp. 2363-2367.

[3] Z. Xiang and M. Tao, "Robust beamforming for wireless information and power transmission," IEEE Commun. Lett., vol. 1, no. 4, pp. 372 375, Aug. 2012.

[4] A. Nasir, X. Zhou, S. Durrani, and R. Kennedy, "Relaying protocols for wireless energy harvesting and information processing," IEEE Trans. Wireless Commun., vol. 12, no. 7, pp. 3622-3636, Jul. 2013.

[5] X. Zhou, R. Zhang, and C. K. Ho, "Wireless information and power transfer: Architecture design and rate-energy tradeoff," IEEE Trans. Commun., vol. 61, no. 11, pp. 4754-4767, Nov. 2013.

[6] C. Huang, R. Zhang, and S. Cui, "Throughput maximization for the Gaussian relay channel with energy harvesting constraints," IEEE J. Sel. Areas Commun., vol. 31, no. 8, pp. 1469-1479, Aug. 2013.

[7] I. Ahmed, A. Ikhlef, R. Schober, and R. K. Mallik, "Power allocation for conventional and buffer-aided link adaptive relaying systems with energy harvesting nodes," IEEE Trans. Wireless Commun., vol. 13, no. 3, pp. 1182-1195, Mar. 2014.

[8] Z. Ding, S. Perlaza, I. Esnaola, and H. Poor, "Power allocation strategies in energy harvesting wireless cooperative networks," IEEE Trans. Wireless Commun., vol. 13, no. 2, pp. 846-860, Feb. 2014.

[9] K. Tutuncuoglu, B. Varan, and A. Yener, "Energy harvesting two-way half-duplex relay channel with decode-and-forward relaying: Optimum power policies," in Int. Conf. on Digital Signal Processing (DSP) 2013.

[10] J. Huang, Q. Li, Q. Zhang, G. Zhang, and J. Qin, "Relay beamforming for amplify-and-forward multi-antenna relay networks with energy harvesting constraint," IEEE Signal Processing Letters, vol. 21, no. 4, pp. 454-458, April 2014.

[11] B. Medepally and N. Mehta, "Voluntary energy harvesting relays and selection in cooperative wireless networks," IEEE Trans. Wireless Commun., vol. 9, no. 11, pp. 3543-3553, Nov. 2010.

[12] B. Chalise, W.-K. Ma, Y. Zhang, H. Suraweera, and M. Amin, "Optimum performance boundaries of OSTBC based AF-MIMO relay system with energy harvesting receiver," Signal Processing, IEEE Transactions on, vol. 61, no. 17, pp. 4199-4213, Sep. 2013.

[13] I. Ahmed, A. Ikhlef, R. Schober, and R. Mallik, "Joint power allocation and relay selection in energy harvesting af relay systems," IEEE Trans. Wireless Commun. Lett., vol. 2, no. 2, pp. 239-242, Apr. 2013.

[14] D. Michalopoulos, H. Suraweera, and R. Schober, "Simultaneous information transmission and wireless energy transfer via selecting one out of two relays," in Int. Symposium on Commun., Control and Signal Processing, May 2014, pp. 318-321.

[15] I. Krikidis, "Simultaneous information and energy transfer in large-scale networks with/without relaying," IEEE Trans. Commun., vol. 62, no. 3, pp. 900-912, Mar. 2014.

[16] H. Chen, Y. Li, J. L. Rebelatto, B. F. U. Filhoand, and B. Vucetic, "Harvest-then-cooperate: wireless-powered cooperative communications." [Online]. Available in arXiv.

[17] S. Atapattu, Y. Jing, H. Jiang, and C. Tellambura, "Relay selection schemes and performance analysis approximations for two-way networks," IEEE Trans. Commun., vol. 61, no. 3, pp. 987-998, Mar. 2013.

[18] Z. Ding and H. V. Poor, "Energy harvesting cooperative networks: Is the max-min criterion still diversity-optimal?" [Online]. Available in arXiv.

[19] I. S. Gradshteyn and I. M. Ryzhik, Table of Integrals, Series and Products, 7th ed. Academic Press Inc, 2007.

[20] S. Atapattu, Y. Jing, H. Jiang, and C. Tellambura, "Relay selection and performance analysis in multiple-user networks," IEEE J. Select. Areas Commun., vol. 31, no. 8, pp. 1517-1529, Aug. 2013.

[21] Z. Wang and G. B. Giannakis, "A simple and general parameterization quantifying performance in fading channels," IEEE Trans. Commun., vol. 51, no. 8, pp. 1389-1398, Aug. 2003.

[22] C. B. Boyer and U. C. Merzbach, A History of Mathematics, 2nd ed. New York: Wiley, 1991. 